\title{
The Essential Singularity of the Solution of a Ramified Characteristic Cauchy Problem
}

\author{
By \\ Hideshi YAMANE*
}

\section{Introduction}

J. Leray [L] and L. Gårding, T. Kotake and J. Leray [G-K-L] have studied the singularities of the solution of a Cauchy problem with holomorphic data, when the initial surface includes some characteristic points. They have proved that the solution may be ramified around a hypersurface $K$.

Y. Hamada $[\mathrm{H}]$ has studied another class of characteristic Cauchy problem. In his case, the solution may have an essential singularity, although the data are regular.

Let $P u=v$ be our equation. We already know that we must allow $u$ to be ramified or to have an essential singularity. Now that we understand this necessity, it would be desirable to allow $v$ to be singular without introducing a larger class for $u$.

[D] and $[\mathrm{O}-\mathrm{Y}]$ are studies in this direction. They are generalizations of [L] and $[\mathrm{G}-\mathrm{K}-\mathrm{L}]$.

In the present paper, we consider a problem similar to the one in $[\mathrm{H}]$. Although we impose a stronger condition on the operator $P$ than in $[\mathrm{H}]$, we assume a weaker condition on $v$ : it is allowed to be singular. Moreover, by employing a symbol calculus like the one in [D], we can explain easily why $u$ has an essential singularity even for a holomorphic $v$.

\section{§1. Statement of the Results}

Let $S$ and $K$ be the hypersurfaces in $\mathbb{C}_{x}^{n}$ defined by $x_{1}=x_{2}^{q}$ and $x_{1}=0$ respec-

Communicated by T. Kawai, November 27, 1995. Revised January 22, 1996.

1991 Mathematics Subject Classification. Primary 35A20; Secondary 35S99.

* Department of Mathematical Sciences, University of Tokyo

3-8-1, Komaba, Meguro-ku, Tokyo 153, Japan.

Current address: Department of Mathematics, Chiba Institute of Technology, 2-1-1 Shibazono, Narashino, Chiba 275, Japan. 
tively, where $q$ is an integer $\geq 2$. We introduce a class of the stalk of ramified functions at $x=0$, denoted by $\mathcal{N}_{q, K}$. It is defined by

$$
f(x) \in \mathcal{N}_{q, K} \Longleftrightarrow f(x)=\sum_{j=0}^{q-1} f_{j}(x) x_{1}^{j / q}, \quad f_{j} \text { is holomorphic near } x=0 .
$$

We set

$$
\mathcal{N}_{q, K}^{l}=\left\{f(x) \in \mathcal{N}_{q, K} ; \quad f \quad \text { vanishes on } S \text { up to order } l\right\} \quad(l \geq 0)
$$

Moreover, we set

$$
\widetilde{\mathcal{N}}_{q, K}=\sum_{j=0}^{q-1} x_{1}^{j / q} \underset{X \ni 0}{\lim } \mathfrak{O}(X \backslash K)
$$

A function in $\widetilde{\mathcal{N}}_{q, K}$ may be ramified and have an essential singularity.

To formulate a Cauchy problem, we introduce

$$
\widetilde{\mathcal{N}}_{q, K}^{l}=\left\{f \in \widetilde{\mathcal{N}}_{q, K} ; \quad f \text { vanishes on } S \text { up to order } l\right\} \quad(l \geq 0) .
$$

We have

Theorem 1. Let $P(x, D)$ be a differential operator near the origin

$$
P(x, D)=D_{1}^{A_{1}} D_{2}^{A_{2}}-\sum_{|\alpha|<A_{1}+A_{2}} D^{\alpha} a_{\alpha}(x), \quad A_{1} \geq 0, A_{2} \geq 0
$$

where $a_{\alpha}(x)$ is holomorphic near the origin and is a polynomial in $x_{1}$ and $x_{2}$. Then, for any element $v(x)$ of $D_{1}^{A_{1}} \mathcal{N}_{q, K}^{A_{1}}$, there exists a unique element $u(x)$ of $\widetilde{\mathcal{N}}_{q, K}^{A_{1}+A_{2}}$ such that

$$
P u=v
$$

holds.

Remark. If $\sum_{|\alpha|<A_{1}+A_{2}} D^{\alpha} a_{\alpha}(x)$ is of order less than $A_{1}$ with respect to $D_{1}$, then $P$ belongs to the class treated in $[\mathrm{O}-\mathrm{Y}]$ and the solution $u$ is in $\mathcal{N}_{q, K}^{A_{1}+A_{2}}$.

Theorem 2. ([O-Y]) Assume that $A_{1} \geq 1$. Then
(A)
$x_{1}^{-\frac{a-1}{q}} \mathcal{N}_{q, K} \subset D_{1}^{A_{1}} \mathcal{N}_{q, K}^{A_{1}} . \quad$ Equality holds if $A_{1}=1$. 
(B) $\quad x_{1}^{-\frac{1}{q}} \notin D_{1}^{A_{1}} \mathcal{N}_{q, K}^{A_{1}}$ if $l \geq q$.

The proof of Theorem 2 is given in $[\mathrm{O}-\mathrm{Y}]$. In the following, we are going to prove Theorem 1 .

\section{§2. The Inverse of a Microdifferential Operator}

We review the definition of microdifferential operators and formal norms. For details, see $[\mathrm{K}-\mathrm{K}-\mathrm{K}]$.

Definition 1. Let $\Omega$ be a conic open set of $T^{*} \mathbf{C}_{x}^{n}$. We denote by $\xi$ the dual variable of $x$. Let $P(x, \xi)$ be a formal sum of the following form:

$$
P(x, \xi)=\sum_{k=0}^{\infty} p_{m-k}(x, \xi)
$$

where $p_{m-k}(x, \xi)$ is holomorphic in $\Omega$ and is homogeneous of degree $m-k$ with respect to $\xi$. Then $P(x, \xi)$ is said to be a microdifferential operator of order $m$ in $\Omega$ if it satisfies the following growth condition:

For an arbitrary compact subset $K$ in $\Omega$, there exists a positive constant $C_{K}$ such that

$$
\left|p_{m-k}(x, \xi)\right| \leq C_{K}^{k+1} k !
$$

We sometimes write $P(x, \xi)$ as $P(x, D)$.

The correspondence

$\Omega \mapsto\{P(x, D) ; \quad P$ is a microdifferential operator of order $m$ in $\Omega\}$

forms a sheaf on $T^{*} \mathbf{C}^{n}$, which we denote by $\mathscr{E}(m)$.

In the calculus of microdifferential operators, formal norms defined in [Bou-Kr] are very useful.

Definition 2. In the situation of Definition 1 , the formal norm $N_{m}^{K}(P ; t)$ is a formal sum defined as

$$
N_{m}^{K}(P ; t)=\sum_{k, \alpha, \beta} \frac{2(2 n)^{-k} k !}{(|\alpha|+k) !(|\beta|+k) !} \sup _{K}\left|D_{x}^{\alpha} D_{\xi}^{\beta} p_{m-k}(x, \xi)\right| t^{2 k+|\alpha+\beta|}
$$


where the sum is taken with respect to $k \in \mathbb{N}_{0}=\{0,1,2, \ldots\}, \quad \alpha, \beta \in \mathbb{N}_{0}^{n}$.

Remark. If $N_{m}^{K}(P ; \varepsilon)<\infty$ holds for some $\varepsilon>0$, then the growth condition (G) is satisfied. Conversely, if (G) is satisfied, then $N_{m}^{K^{\prime}}(P ; \varepsilon)<\infty$ for some $K^{\prime} \subset K$ and $\varepsilon>0$.

We quote two lemmas from [Y].

Lemma 1. (Lemma 10 of $[\mathrm{Y}]$ ) Let $R(x, D)$ be a microdifferential operator of order $\leq-j<0$ defined in a neighborhood of a compact set $\omega \subset T^{*} \mathbb{C}_{x}^{n}$, where $j$ is a positive integer. Then we have

$$
N_{0}^{\omega}(R ; t) \ll \frac{(2 n)^{-j}}{j !} t^{2 j} N_{-j}^{\omega}(R ; t)
$$

Proof. By definition,

$$
N_{0}^{\omega}(R ; t)=\sum_{k, \alpha, \beta} \frac{2(2 n)^{-k} k !}{(|\alpha|+k) !(|\beta|+k) !} \sup _{\omega}\left|D_{x}^{\alpha} D_{\xi}^{\beta} r_{-k}(x, \xi)\right| t^{2 k+|\alpha+\beta|},
$$

where $R=\sum_{k \geq 0} r_{-k}$ and $r_{-k}$ is the homogeneous part of degree $-k$. There is no contribution by the terms corresponding to $k=0,1,2, \ldots, j-1$. Hence, if we put $l=k-j$,

$$
\begin{aligned}
N_{0}^{\omega}(R ; t)= & \sum_{l \geq 0, \alpha, \beta} \frac{2(2 n)^{-(l+j)}(l+j) !}{(|\alpha|+l+j) !(|\beta|+l+j) !} \\
& \quad \times \sup _{\omega}\left|D_{x}^{\alpha} D_{\xi}^{\beta} r_{-(l+j)}(x, \xi)\right| t^{2(l+j)+|\alpha+\beta|} .
\end{aligned}
$$

We have only to prove that

$$
\frac{2(2 n)^{-(l+j)}(l+j) !}{(|\alpha|+l+j) !(|\beta|+l+j) !} \leq \frac{(2 n)^{-j}}{j !} \frac{2(2 n)^{-l} l !}{(|\alpha|+l) !(|\beta|+l) !}
$$

This inequality is obtained by the calculation below.

$$
\begin{gathered}
\frac{2(2 n)^{-(l+j)}(l+j) !}{(|\alpha|+l+j) !(|\beta|+l+j) !} \times \frac{(|\alpha|+l) !(|\beta|+l) !}{2(2 n)^{-l} l !} \\
\leq(2 n)^{-j} \times \frac{1}{(|\alpha|+l+j) \cdots(|\alpha|+l+1)} \times \frac{(l+j) \cdots(l+1)}{(|\beta|+l+j) \cdots(|\beta|+l+1)} \\
\leq(2 n)^{-j} \times \frac{1}{j !} \times 1 .
\end{gathered}
$$


Lemma 2. (A special case of Lemma 11 of $[\mathrm{Y}]$ ) Let $Q$ be a microdifferential operator of order $\leq-1$. Then we have

$$
\left.N_{0}^{\omega}\left(Q^{j} ; t\right) \ll \frac{(2 n)^{-j}}{j !} t^{2 j}\left\{N_{-1}^{\omega} Q ; t\right)\right\}^{j}
$$

Proof. By $[\mathrm{B}-\mathrm{Kr}]$, we have $N_{-j}^{\omega}\left(Q^{j}\right) \ll\left\{N_{-1}^{\omega}(Q)\right\}^{j}$. Lemma 2 follows from Lemma 1.

Now let us consider $P$ in Theorem 1. Define a microdifferential operator $\widetilde{P}(x, D)$ by

$$
\widetilde{P}(x, D)=D_{1}^{-A_{1}} D_{2}^{-A_{2}} P(x, D)
$$

Obviously we have

$$
\widetilde{P}=1-\sum_{|\alpha|<\mathrm{A}_{1}+A_{2}} D_{1}^{-A_{1}} D_{2}^{-A_{2}} D^{\alpha} a_{\alpha}(x)
$$

and its adjoint $\widetilde{P}^{*}$ is given by

$$
\widetilde{P} *(x, D)=1-\sum_{|\alpha|<A_{1}+A_{2}} a_{\alpha}(x)\left(-D_{1}\right)^{-A_{1}}\left(-D_{2}\right)^{-A_{2}}(-D)^{\alpha}
$$

The summation is of order $\leq-1$. The inverse of $\widetilde{P} *$, which we denote by $R$, is calculated in terms of Neumann series:

$$
R=\left(\widetilde{P}^{*}\right)^{-1}=\sum_{j=0}^{\infty} Q(x, D)^{j}
$$

where

$$
Q(x, D)=\sum_{|\alpha|<A_{1}+A_{2}} a_{\alpha}(x)\left(-D_{1}\right)^{-A_{1}}\left(-D_{2}\right)^{-A_{2}}(-D)^{\alpha} \in \mathscr{E}(-1)
$$

Let $q_{j k}$ be the homogeneous term of degree $(-k)$ of $Q^{j}$ : i.e.

$$
Q(x, D)^{j}=\sum_{k=j}^{\infty} q_{j k}(x, D) \in \mathscr{E}(-j)
$$


In fact, this is a finite sum as we will see later). By lemma 2 and the definition of the formal norm, we have

$$
\frac{2(2 n)^{-k} t^{2 k}}{k !} \sup \left|q_{j k}\right| \leq \frac{(2 n)^{-j}}{j !} t^{2 j}\left\{N_{-1}(Q ; t)\right\}^{j} \quad \text { if } t>0
$$

(For simplicity, we neglect to specify a compact set). Hence

$$
\left|q_{j k}\right| \leq \frac{1}{2}(2 n)^{-j+k} \frac{k !}{j !} t^{2(j-k)}\left\{N_{-1}(Q ; t)\right\}^{j} .
$$

Next, we show the above-mentioned fact that $Q^{j}=\sum_{k} q_{j k}$ is a finite sum. In fact, we have

Lemma 3. There exists a positive integer $m$ independent of $j$ such that $Q^{j}$ consists of homogeneous terms of degree $-j,-(j+1), \cdots,-m j$.

Proof. A term of the form $a(x) D_{1}^{\gamma_{1}} D_{2}^{\gamma_{2}} \cdots D_{n}^{\gamma_{n}}$ is said to be of type $(s,-t)$, $s \in \mathbb{N}_{0}, t \in \mathbb{N}_{0}=\{0,1,2,3, \cdots\}$, where $a$ is a holomorphic function which is a polynomial in $x_{1}$ and $x_{2}$ of degree $\leq s$ and $\gamma_{1}+\cdots+\gamma_{n} \geq-t, \gamma_{1} \in \mathbb{Z}, \gamma_{2} \in \mathbb{Z}$, $\gamma_{3} \in \mathbb{N}_{0}, \ldots, \gamma_{n} \in \mathbb{N}_{0}$. (If $s^{\prime} \geq s$ and $t^{\prime} \geq t$, then a term of type $(s,-t)$ is of type $\left.\left(s^{\prime},-t^{\prime}\right)\right)$.

Let $a_{\alpha}$ 's be polynomials in $x_{1}$ and $x_{2}$ of degree $\leq l$. Then $Q$ consists of terms of type $(l,-A), A=A_{1}+A_{2}$.

It is easy to see that if $r_{1}(x, D)$ (resp. $\left.r_{2}(x, D)\right)$ is of type $\left(s_{1},-t_{1}\right)$ (resp. $\left.\left(s_{2},-t_{2}\right)\right)$, then $r_{1}(x, D) r_{2}(x, D)$ consists of terms of type $\left(s_{1}+s_{2},-t_{1}-t_{2}\right)$, $\left(s_{1}+s_{2}-1,-t_{1}-t_{2}-1\right), \cdots,\left(0,-s_{1}-s_{2}-t_{1}-t_{2}\right)$.

By induction, we can prove that $Q^{j}$ consists of terms of type $(j l,-j A), \cdots$, $(0,-j l-j A)$. Combining this with the fact that ord $Q^{j} \leq-j$, we obtain the lemma.

Let $r_{k}(x, D)$ be the homogeneous term of degree $(-k)$ of the operator $R(x, D)=\widetilde{P}^{*}(x, D)^{-1}=\sum_{j=0}^{\infty} Q(x, D)^{j}$. Then $R=\sum_{k=0}^{\infty} r_{k}(x, D)$ and, by the lem ma above,

$$
r_{k}=\sum_{j=\left\lceil\frac{k}{m}\right\rceil}^{k} q_{j k}, \quad \text { where }\left\lceil\frac{k}{m}\right\rceil=\min \left\{n \in \mathbf{N}_{0} ; n \geq \frac{k}{m}\right\}
$$

We employ the estimate (1) to obtain

$$
\left|r_{k}\right| \leq \sum_{j=\left\lceil\frac{k}{m}\right\rceil}^{k}\left|q_{j k}\right| \leq \sum_{j=\left\lceil\frac{k}{m}\right\rceil}^{k} \frac{1}{2}(2 n)^{-j+k} \frac{k !}{j !} t^{2(j-k)}\left\{N_{-1}(Q ; t)\right\}^{j} .
$$


By using

$$
\frac{1}{j !} \leq \frac{1}{\left\lceil\frac{k}{m}\right\rceil !\left(j-\left\lceil\frac{k}{m}\right\rceil\right) !}
$$

we see that

$$
\begin{aligned}
\left|r_{k}\right| \leq & \frac{1}{2}(2 n)^{k} k ! \frac{1}{\left\lceil\frac{k}{m}\right\rceil !} t^{-2 k}(2 n)^{-\left\lceil\frac{k}{m}\right\rceil}\left\{t^{2} N_{-1}(Q ; t)\right\}^{\left\lceil\frac{k}{m}\right\rceil} \\
& \times \sum_{j=\left\lceil\frac{k}{m}\right\rceil}^{k}(2 n)^{-\left(j-\left\lceil\frac{k}{m}\right\rceil\right)} \frac{1}{\left(j-\left\lceil\frac{k}{m}\right\rceil\right) !}\left\{t^{2} N_{-1}(Q ; t)\right\}^{j-\left\lceil\frac{k}{m}\right\rceil} \\
\leq & \frac{1}{2}(2 n)^{k} k ! \frac{1}{\left\lceil\frac{k}{m}\right\rceil !} t^{-2 k}(2 n)^{-\left\lceil\frac{k}{m}\right\rceil}\left\{t^{2} N_{-1}(Q ; t)\right\}^{\left\lceil\frac{k}{m}\right\rceil} \\
& \cdot \exp \left\{\frac{1}{2 n} t^{2} N_{-1}(Q ; t)\right\} .
\end{aligned}
$$

Therefore, for any compact set $\omega$ of $\left\{x \in \mathbf{C}^{n} ;|x| \ll 1\right\} \times\left\{\xi ; \xi_{1} \neq 0, \xi_{2} \neq 0\right\} \subset T^{*} \mathbf{C}_{x}^{n}$, there exists a positive constant $C_{\omega}$ independent of $k$ such that

$$
\sup _{\omega}\left|r_{k}(x, \xi)\right| \leq C_{\omega}^{k+1} \frac{k !}{\left\lceil\frac{k}{m}\right\rceil !}
$$

Here $|x| \ll 1$ means that $|x|$ is sufficiently small. Now set

$$
r_{k}(x, D)=\sum_{|\beta|=-k} b_{\beta}(x) D^{\beta} \in \mathscr{E}\left(\{|x| \ll 1\} \times\left\{\xi_{1} \neq 0, \xi_{2} \neq 0\right\}\right) .
$$

Let us obtain an estimate on $b_{\beta}(x)$ when $\beta_{1}>0\left(\Rightarrow \beta_{2}<0\right)$. Remark that the partial sum

$$
\sum_{k \geq 0} \sum_{\substack{|\beta|=-k \\ \beta 1 \leq 0}} b_{\beta}(x) D^{\beta}
$$

belongs to the class $\mathscr{E}_{K}$ of $[\mathrm{D}]$, and it is already well understood.

Since

$$
\begin{aligned}
b_{\beta}(x)=\frac{1}{(2 \pi i)^{n-1}} \oint_{\left|\xi_{2}\right|=\delta 2} \oint_{\left|\xi_{3}\right|=\delta^{\prime}} & \cdots \oint_{|\xi n|=\delta^{\prime}} \xi_{2}^{-\beta_{2}-1} \xi_{3}^{-\beta_{3}-1} \cdots \xi_{n}^{-\beta_{n}-1} \\
& \times r_{k}\left(x ; 1, \xi_{2}, \xi_{3}, \ldots, \xi_{n}\right) d \xi_{2} d \xi_{3} \cdots d \xi_{n}
\end{aligned}
$$

we obtain, owing to (2) 


$$
\left|b_{\beta}(x)\right| \leq C_{\delta 2, \delta^{\prime}}^{k+1} \frac{k !}{\left\lceil\frac{k}{m}\right\rceil !} \delta_{2}^{-\beta_{2}} \delta^{\prime-\left|\beta^{\prime}\right|}, \beta^{\prime}=\left(\beta_{3}, \ldots, \beta_{n}\right),
$$

where $C_{\delta, \delta^{\prime}}$ is a positive constant independent of $k$.

Before concluding this section, we remark that

$$
\widetilde{P}^{-1}=R^{*}=\sum_{k=0}^{\infty}\left\{\gamma_{k}(x, D)\right\} *=\sum_{k=0}^{\infty} \sum_{|\beta|=-k}(-D)^{\beta} b_{\beta}(x) .
$$

\section{§3. Some Preparation}

\section{Lemma 4.}

$$
\left(\frac{1}{z^{q-1}} D_{z}\right)^{j}=\frac{1}{z^{q j}}\{\theta-q(j-1)\} \cdots\{\theta-q\} \theta, \quad j \geq 1
$$

where $\theta=z D_{z}$.

Proof. One has

$$
\theta \frac{1}{z^{k}}=\frac{1}{z^{k}} \theta-z \frac{k}{z^{k+1}}=\frac{1}{z^{k}}(\theta-k) .
$$

The lemma is proved by induction.

Lemma 5. Let $j$ be a positive integer. We have for $0<y<1$,

$$
\sum_{k=0}^{\infty} \underbrace{\{k+q(j-1)\} \cdots\{k+q\} k y^{k}}_{j \text { factors }} \leq \frac{j ! y^{q}}{(1-y)\left\{y^{q-1}(1-y)\right\}^{j}}
$$

Proof. In fact,

$$
\begin{aligned}
& \sum_{k=0}^{\infty}\{\underbrace{\{k+q(j-1)\} \cdots\{k+q\} k y^{k}}_{j \text { factors }} \\
\leq & \sum_{k=0}^{\infty}\{\underbrace{\{k+q(j-1)\}\{k+q j-q-1\} \cdots\{k+(q-1)(j-1)\}}_{j \text { factors }} y^{k} \\
= & \frac{1}{y^{q j-q-j}} \frac{d^{j}}{d y^{j}} \sum_{k=0}^{\infty} y^{k+q(j-1)}
\end{aligned}
$$




$$
\begin{aligned}
& \leq \frac{y^{q}}{y^{(q-1) j}} \frac{d^{j}}{d y^{j}}\left(1+y+y^{2}+\cdots\right) \\
& =\frac{y^{q}}{y^{(q-1) j}} \frac{j !}{(1-y)^{j+1}}
\end{aligned}
$$

Lemma 6. Let $f(z)$ be a holomorphic function in $\{z \in \mathbf{C} ;|z|<r+\varepsilon\}, r>0$, $\varepsilon>0$. If $|f(z)| \leq M$ holds in $\{z \in \mathbb{C} ;|z| \leqq r\}$ then we have, in $\{z \in \mathbb{C} ; 0<|z|<r\}$,

$$
\left|\left(\frac{1}{z^{q-1}} D_{z}\right)^{j} f(z)\right| \leq M \frac{j !\left(\frac{|z|}{r}\right)^{q}}{\left(1-\frac{|z|}{r}\right)\left\{|z|^{q}\left(\frac{|z|}{r}\right)^{q-1}\left(1-\frac{|z|}{r}\right)\right\}^{j}} .
$$

Proof. Let the Taylor expansion of $f$ be

$$
f(z)=\sum_{k=0}^{\infty} f_{k} z^{k}
$$

Then we have

$$
f_{k}=\frac{1}{2 \pi i} \oint_{|z|=r} \frac{f(z)}{z^{k+1}} d z, \quad\left|f_{k}\right| \leq M r^{-k}
$$

By using Lemma 4 we see that

$$
\left(\frac{1}{z^{q-1}} D_{z}\right)^{j} f(z)=\sum_{k=0}^{\infty} f_{k} \frac{1}{z^{q j}}\{k-q(j-1)\} \cdots\{k-q\} k z^{k}
$$

The series in the right hand side is estimated by Lemma 5 . We obtain

$$
\begin{aligned}
& \left|\left(\frac{1}{z^{q-1}} D_{z}\right)^{j} f(z)\right| \\
\leq & \sum_{k=0}^{\infty} M r^{-k} \frac{1}{|z|^{q j}}\{k+q(j-1)\} \cdots\{k+q\} k|z|^{k} \\
= & \frac{M}{|z|^{\mid q j}} \frac{j !\left(\frac{|z|}{r}\right)^{q}}{\left(1-\frac{|z|}{r}\right)\left\{\left(\frac{|z|}{r}\right)^{q-1}\left(1-\frac{|z|}{r}\right)\right\}^{j}} \cdot
\end{aligned}
$$




\section{§4. The Action of a Microdifferential Operator on a Ramified Function}

For the study of $\mathcal{N}_{q, K}$, we introduce a singular coordinate change $z=x_{1}^{1 / q}$. We denote by $\widetilde{S}$ the hypersurface of $\mathbb{C}_{z, x_{2}, x^{\prime}}^{n}$ defined by $z=x_{2}$. Here $x^{\prime}=\left(x_{3}, \cdots, x_{n}\right)$. The singular coordinate change induces an isomorphism

$$
\begin{gathered}
\mathcal{N}_{q, K} \simeq \mathscr{O}_{\left(z, x, x^{\prime}\right)=0} \\
f(x)=\sum_{j=0}^{q-1} f_{j}(x) x_{1}^{j / q} \mapsto \widetilde{f}\left(z, x_{2}, x^{\prime}\right)=\sum_{j=0}^{q-1} f_{j}\left(z^{q}, x_{2}, x^{\prime}\right) z^{j} .
\end{gathered}
$$

Moreover $f \in \mathcal{N}_{q, K}^{l}$ if and only if $\widetilde{f}$ vanishes on $\widetilde{S}$ up to order $l$.

Proposition 1. ([D]) Proposition 6) The characteristic Cauchy problem

$$
D_{2} 2=f \in \mathcal{N}_{q, K}^{l}
$$

admits a unique solution $g \in \mathcal{N}_{q, K}^{l+1}$. Moreover, if we have

$$
\left|\widetilde{f}\left(z, x_{2}, x^{\prime}\right)\right| \leq M\left\{|z|+\left|x_{2}-z\right|\right\}^{m}
$$

for some positive constant $M$ and a non-negative integer $m$, then

$$
\left|\widetilde{g}\left(z, x_{2}, x^{\prime}\right)\right| \leq \frac{M}{m+1}\left\{|z|+\left|x_{2}-z\right|\right\}^{m+1} .
$$

Proof. The equation $D_{2} g=f$ is equivalent to $D_{2} \widetilde{g}=\widetilde{f}$, and the initial surface $S$ is transformed into $\widetilde{S}$. Since $\widetilde{S}$ is noncharacteristic, we can find a unique holomorphic solution $\widetilde{g}$. The estimate is obtained by an elementary integral representation.

This proposition suggests that $\mathcal{N}_{q, K}$ and its variants are more suitable classes for the study of characteristic Cauchy problems than that of holomorphic functions.

Definition 3. We can define

$$
D_{2}^{-1}: \mathcal{N}_{q, K}^{l} \rightarrow \mathcal{N}_{q, K}^{l+1}
$$

by using the proposition above. It is a right inverse of 


$$
D_{2}: \mathcal{N}_{q, K}^{l+1} \rightarrow \mathcal{N}_{q, K}^{l}
$$

but it is not a left inverse.

Remark. If $u$ is an element of $\mathcal{N}_{q, K}$ and $f$ is holomorphic near $x=0$, then we can define $D_{2}^{-l}(f(x) u(x)), l \in \mathbf{N}_{0}$. It is the unique solution of the Cauchy problem

$$
\left\{\begin{array}{l}
D_{2}^{l} w(x)=f(x) u(x) \\
w(x) \in \mathcal{N}_{q, K}^{l}
\end{array}\right.
$$

On the other hand, $D_{2}^{-l} \circ f(x)$ belongs to the symbol class $\mathscr{E}_{K}$ in [D], and $\left(D_{2}^{-l} \circ f(x)\right) u(x) \in \mathcal{N}_{q, K}^{l}$ is defined in [D]. Dunau puts integration on the right:

$$
D_{2}^{-l} \circ f(x)=f(x) D_{2}^{-l}+\sum_{j=l+1}^{\infty} f_{j}(x) D_{2}^{-j}
$$

for some $f_{j}(x)$. He sets

$$
\left(D_{2}^{-l} \circ f(x)\right) u(x) \underset{\operatorname{def}}{=} f(x) D_{2}^{-l} u(x)+\sum_{j=l+1}^{\infty} f_{j}(x) D_{2}^{-j} u(x)
$$

It satisfies the same equation as above and we see that

$$
D_{2}^{-l}(f(x) u(x))=\left(D_{2}^{-l} \circ f(x)\right) u(x)
$$

So it makes no difference whether integration comes on the left or on the right.

Now we are ready to define $\widetilde{P}(x, D)^{-1} w(x) \in \widetilde{\mathcal{N}}_{q, K}$, where $\widetilde{P}$ is as in the second section and $w(x) \in \mathcal{N}_{q, K}$.

$\widetilde{P}^{-1}$ has the expression

$$
\widetilde{P}^{-1}=\sum_{k=0}^{\infty} \sum_{|\boldsymbol{\beta}|=-k}(-D)^{\beta} b_{\beta}(x) \in \mathscr{E}\left(\left\{|x| \ll 1, \xi_{1} \neq 0, \xi_{2} \neq 0\right\}\right), \text { ord } \widetilde{P}^{-1} \leq 0
$$

The partial sum consisting of the terms corresponding to $\beta_{1} \leq 0$ belongs to Dunau's class $\mathscr{E}_{K}$ and its action on $\mathcal{N}_{q, K}$ is defined in [D]. Therefore, in order to define the action of $\widetilde{P}^{-1}$, we may assume without loss of generality that $b_{\beta} \equiv 0$ if $\beta_{1} \leq 0$. This means that $\beta_{2}<0$ in the sum. 
We set

$$
\widetilde{P}^{-1}(x, D) w(x)=\sum_{k=0}^{\infty} \sum_{|\beta|=-k}(-D)^{\beta} b_{\beta}(x) w(x)
$$

We are going to prove that it defines an element of $\widetilde{\mathcal{N}}_{q, K}$. Put $x_{1}^{1 / q}=z$, $\widetilde{w}\left(z, x_{2}, x^{\prime}\right)=w\left(z^{q}, x_{2}, x^{\prime}\right)$, and $\widetilde{b}_{\beta}\left(z, x_{2}, x^{\prime}\right)=b_{\beta}\left(z^{q}, x_{2}, x^{\prime}\right)$. Then

$$
\begin{aligned}
\left(\widetilde{P}^{-1} w\right)(x)= & \sum_{k=0}^{\infty} \sum_{|\beta|=-k}\left(\frac{1}{q z^{q-1}} D_{z}\right)^{\beta_{1}} D_{2}^{\beta_{2}} D^{\prime \beta^{\prime}} \\
& \cdot(-1)^{|\beta|} \widetilde{b}_{\beta}\left(z, x_{2}, x^{\prime}\right) \widetilde{w}\left(z, x_{2}, x^{\prime}\right) .
\end{aligned}
$$

(3) in the second section implies that in a neighborhood $X$ of $\left(z, x_{2}, x^{\prime}\right)=0$, we have

$$
\left|(-1)^{|\beta|} \widetilde{b}_{\beta} \widetilde{w}\right| \leq C_{\delta_{2}, \delta^{\prime}}^{k+1} \cdot \frac{k !}{\left\lceil\frac{k}{m}\right\rceil !} \delta_{2}^{-\beta_{2}} \delta^{\prime-\left|\beta^{\prime}\right|} \sup _{X}|\widetilde{w}|, \quad|\beta|=-k
$$

In a smaller neighborhood, there exists a positive constant $r^{\prime}>0$ such that

$$
\left|D^{\prime \beta^{\prime}} \cdot(-1)^{|\beta|} \widetilde{b}_{\beta} \widetilde{w}\right| \leq \beta^{\prime} ! r^{\prime-\left|\beta^{\prime}\right|} C_{\delta_{2}, \delta^{\prime}}^{k+1} \cdot \frac{k !}{\left\lceil\frac{k}{m}\right\rceil !} \delta_{2}^{-\beta_{2}} \delta^{\prime-\left|\beta^{\prime}\right|} \sup _{X}|\widetilde{w}| .
$$

Then, we employ Proposition 1 repeatedly, first for $m=0$, next for $m=1$ and so on. We obtain

$$
\left|D_{2}^{\beta_{2}} D^{\prime \beta^{\prime}} \cdot(-1)^{|\beta|} \widetilde{b}_{\beta} \widetilde{w}\right| \leq \frac{\lambda^{-\beta_{2}}}{\left(-\beta_{2}\right) !} \beta^{\prime !} ! r^{\prime-\left|\beta^{\prime}\right|} C_{\delta 2, \delta^{\prime}}^{k+1} \cdot \frac{k !}{\left\lceil\frac{k}{m}\right\rceil !} \delta_{2}^{-\beta_{2}} \delta^{\prime-\left|\beta^{\prime}\right|} \sup _{X}|\widetilde{w}|
$$

in $\left\{|z|<\lambda / 3,\left|x_{2}\right|<\lambda / 3, \ldots,\left|x_{n}\right|<\lambda / 3\right\}$.

By using Lemma 6, we see that

$$
\begin{aligned}
& \left|\left(\frac{1}{q z^{q-1}} D_{z}\right)^{\beta_{1}} D_{2}^{\beta_{2}} D^{\beta^{\prime}} \cdot(-1)^{|\beta|} \widetilde{b}_{\beta} \widetilde{w}\right| \\
& \leq \frac{\beta_{1} !\left(\frac{|z|}{r}\right)^{q}}{\left(1-\frac{|z|}{r}\right)\left\{q|z|^{q}\left(\frac{|z|}{r}\right)^{q-1}\left(1-\frac{|z|}{r}\right)\right\}^{\beta_{1}}} \\
& \quad \times \frac{\lambda^{-\beta_{2}}}{\left(-\beta_{2}\right) !} \beta^{\prime} ! r^{\prime-\left|\beta^{\prime}\right|} C_{\delta 2, \delta^{\prime}}^{k+1} \cdot \frac{k !}{\left\lceil\frac{k}{m}\right\rceil !} \delta_{2}^{-\beta_{2}} \delta^{\prime-\left|\beta^{\prime}\right|} \sup _{X}|\widetilde{w}|
\end{aligned}
$$


in $\left\{0<|z|<r<\lambda / 3,\left|x_{2}\right|<\lambda / 3, \ldots,\left|x_{n}\right|<\lambda / 3\right\}$.

There exists a constant $C_{z}>1$ depending continuously on $|z|, 0<|z|<r$, such

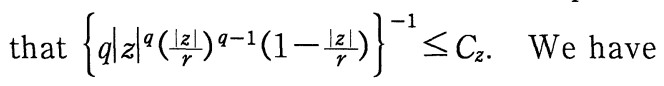

$$
\frac{1}{\left\{q|z|^{q}\left(\frac{|z|}{r}\right)^{q-1}\left(1-\frac{|z|}{r}\right)\right\}^{\beta_{1}}} \leq C_{z}^{\beta_{1}} \leq C_{z}^{\beta_{1}+\left|\beta^{\prime}\right|+k}=C_{z}^{-\beta_{2}} .
$$

Moreover, if we take $\delta^{\prime}>0$ so small that $r^{\prime} \delta^{\prime}<1$, then

$$
\left(r^{\prime} \delta^{\prime}\right)^{-\left|\beta^{\prime}\right|} \leq\left(r^{\prime} \delta^{\prime}\right)^{-\left|\beta^{\prime}\right|-\beta_{1}-k}=\left(r^{\prime} \delta^{\prime}\right)^{\beta_{2}} .
$$

In addition, it is easy to see that

$$
\frac{\beta_{1} ! \beta^{\prime} ! k !}{\left(-\beta_{2}\right) !} \leq 1
$$

because $\beta_{1}+\left|\beta^{\prime}\right|+k=-\beta_{2}$. Combining (4) with these three inequalities, we obtain

$$
\begin{aligned}
& \left|\left(\frac{1}{q z^{q-1}} D_{z}\right)^{\beta 1} D_{2}^{\beta_{2}} D^{\prime \beta^{\prime}} \cdot(-1)^{|\beta|} \widetilde{b}_{\beta} \widetilde{w}\right| \\
& \quad \leq \frac{\left(\frac{|z|}{r}\right)^{q}}{\left(1-\frac{|z|}{r}\right) \cdot\left\lceil\frac{k}{m}\right\rceil !}\left(\frac{C_{z} \lambda \delta_{2}}{r^{\prime} \delta^{\prime}}\right)^{-\beta 2} C_{\delta 2, \delta^{\prime}}^{k+1} \sup _{X}|\widetilde{w}| .
\end{aligned}
$$

For fixed $k$ and $\beta_{2}$, we have

$$
\#\left\{\left(\beta_{1}, \beta^{\prime}\right) ; \beta_{1}>0, \beta^{\prime} \in \mathbb{N}_{0}^{n-2}, \beta_{1}+\beta_{2}+\left|\beta^{\prime}\right|=-k\right\} \leq 2^{n-2-k-\beta_{2}} .
$$

Hence,

$$
\begin{aligned}
& \left|\sum_{k \geq 0} \sum_{|\beta|=-k}\left(\frac{1}{q z^{q-1}} D_{z}\right)^{\beta_{1}} D_{2}^{\beta_{2}} D^{\prime \beta^{\prime}} \cdot(-1)^{|\beta|} \widetilde{b}_{\beta} \widetilde{w}\right| \\
\leq & \frac{\left(\frac{|z|}{r}\right)^{q} \sup _{X}|\widetilde{w}|}{1-\frac{|z|}{r}} \sum_{k \geq 0} \frac{C_{\delta 2, \delta^{\prime}}^{k+1}}{\left[\frac{k}{m}\right] !} \sum_{\beta_{2}=-\infty}^{-1} \sum_{\beta_{1}+\left|\beta^{\prime}\right|=-k-\beta_{2}}\left(\frac{C_{z} \lambda \delta_{2}}{r^{\prime} \delta^{\prime}}\right)^{-\beta_{2}} \\
\leq & \frac{2^{n-2}\left(\frac{|z|}{r}\right)^{q} \sup _{X}|\widetilde{w}|}{1-\frac{|z|}{r}} \sum_{k \geq 0} \frac{2^{-k} C_{\delta 2, \delta^{\prime}}^{k+1}}{\left\lceil\frac{k}{m}\right\rceil !} \sum_{\beta_{2}=-\infty}^{-1}\left(\frac{2 C_{2} \lambda \delta_{2}}{r^{\prime} \delta^{\prime}}\right)^{-\beta_{2}}
\end{aligned}
$$


The right hand side converges on every compact set of $\left\{0<|z| \ll 1,\left|x_{2}\right| \ll 1\right.$, $\left.\ldots,\left|x_{n}\right| \ll 1\right\}$ if we take a sufficiently small $\delta_{2}>0$ in accordance with the compact set.

Summing up, we have finally proved that

$$
\left(\widetilde{P}^{-1} w\right)(x) \in \widetilde{\mathcal{N}}_{q, K}
$$

Moreover, if $w \in \mathcal{N}_{q, K}^{A_{1}+A_{2}}$, then it is easy to see that

$$
\left(\widetilde{P}^{-1} w\right)(x) \in \widetilde{\mathcal{N}}_{q, K}^{A_{1}+A_{2}}
$$

\section{§5. Proof of Theorem 1}

First, remark that

$$
D_{2}^{A_{2}}: \mathcal{N}_{q, K}^{A_{1}+A_{2}} \stackrel{\sim}{\longrightarrow} \mathcal{N}_{q, K}^{A_{1}}
$$

Hence

$$
D_{1}^{A_{1}} \mathcal{N}_{q, K}^{A_{1}}=D_{1}^{A_{1}} D_{2}^{A_{2}} \mathcal{N}_{q, K}^{A_{1}+A_{2}}
$$

Let us solve $P u=D_{1}^{A_{1}} D_{2}^{A_{2}} w, w \in \mathcal{N}_{q, K}^{A_{1}+A_{2}}$. The solution $u$ is given by $u=$ $\widetilde{P}^{-1} w \in \widetilde{\mathcal{N}}_{q, K}^{A_{1}+A_{2}}$. In fact,

$$
P u=P\left(\widetilde{P}^{-1} w\right)=D_{1}^{A_{1}} D_{2}^{A_{2}} w
$$

holds.

The uniqueness is a consequence of Cauchy-Kowalevski theorem, which we apply at noncharacteristic points.

\section{§6. Hamada's Example}

Hamada $([\mathrm{H}])$ gave the following example.

$$
\left\{\begin{array}{l}
\left(D_{2}^{2}-D_{1}\right) u(x)=0 \\
\left.u\right|_{s}=\gamma_{1} x_{2}^{3}, D_{1} u \mid s=\gamma_{2} x_{2}
\end{array}\right.
$$


where

$$
S=\left\{x_{1}=x_{2}^{2}\right\}, \quad \gamma_{1}=\sum_{m=0}^{\infty}(-1)^{m} \frac{\Gamma\left(m-\frac{3}{2}\right)}{(2 m) !}, \quad \gamma_{2}=\sum_{m=0}^{\infty}(-1)^{m+1} \frac{\Gamma\left(m-\frac{1}{2}\right)}{(2 m) !}
$$

The solution $u(x)$ is given by

$$
u(x)=\sum_{m=0}^{\infty}(-1)^{m} \frac{\Gamma\left(m-\frac{3}{2}\right)}{(2 m) !} x_{1}^{\frac{3}{2}-m} x_{2}^{2 m}
$$

It is ramified and has an essential singularity. Let us interpret this phenomenon from our viewpoint. First we reduce the problem to the following one.

$$
\left\{\begin{array}{l}
\left(D_{2}^{2}-D_{1}\right) u(x)=v(x), v \in \mathscr{O}_{x=0} \text { is given, } \\
\left.u\right|_{s}=0,\left.\quad D_{1} u\right|_{s}=0
\end{array}\right.
$$

By using

$$
\begin{aligned}
& \left(D_{2}^{2}-D_{1}\right)^{-1}=\left(1-D_{1} D_{2}^{-2}\right)^{-1} D_{2}^{-2} \\
= & \sum_{j=0}^{\infty}\left(D_{1} D_{2}^{-2}\right)^{j} D_{2}^{-2}=\sum_{j=0}^{\infty} D_{1}^{j} D_{2}^{-2 j-2},
\end{aligned}
$$

we can express the solution by

$$
u(x)=\sum_{j=0}^{\infty} D_{1}^{j} D_{2}^{-2 j-2} v(x)
$$

Put $z=x_{1}^{1 / 2}$. Then we obtain

$$
u\left(z^{2}, x_{2}, x^{\prime}\right)=\sum_{j=0}^{\infty}\left(\frac{1}{2 z} D_{z}\right)^{j} D_{2}^{-2 j-2} v\left(z^{2}, x_{2}, x^{\prime}\right)
$$

Ramification is caused by $D_{2}^{-2 j-2}$. The essential singularity appears because of the factor $\left(\frac{1}{2 z} D_{z}\right)^{j}$. 


\section{References}

[Bou-kr] Boutet de Monvel, L. and Kreé, P., Pseudodifferential operators and Gevrey classes. Ann. Inst. Fourier, 17-1 (1967), 295-323.

[D] Dunau, J., Un Problème de Cauchy Caractéristique, J. Math. pures et appl., 69 (1990), 369-402.

[G-K-L] Gårding, L., Kotake, T. and Leray, J., Problème de Cauchy, I bis et VI, Bull. Soc. Math. France, 92 (1964), 263-361.

[H] Hamada, Y., Les singularités des solutions du problème de Cauchy à données holomorphes, Recent developments in hyperbolic equations (Pisa, 1987), Longman, (1988), 82-95.

[K-K-K] Kashiwara, M., Kawai, T. and Kimura, T., Foundation of Algebraic Analysis, Kinokuniya, 1980 (in Japanese) ; English translation from Princeton, 1986.

[L] Leray, J., Uniformisation de la solution du problème linéaire analytique de Cauchy près de la variété qui porte les données de Cauchy, Bull. Soc. Math. France, 85 (1957) , $389-429$.

[N-S] Nakamura, G. and Sasai, T., The singularities of the solutions of the Cauchy problem for second order equations in case the initial manifold includes characteristic points. Tôhoku Math. J., 28 (1976), 523-539.

[O-Y] Okada, Y. and Yamane, H., A characteristic Cauchy problem in the complex domain, to appear in J. Math. Pures Appl.

[Y] Yamane, H., Branching of singularities for some second or third order microhyperbolic operators., J. Math. Sci. Univ. Tokyo, 2(3) (1996), 1-79. 